\title{
CONOCIMIENTO SOBRE EFECTOS NOCIVOS DEL USO DE AURICULARES PARA MUSICA Y PRACTICAS DE RIESGO PARA LA SALUD EN ESTUDIANTES DE LA FACULTAD DE ENFERMERIA - UNICA SETIEMBRE 2012 - AGOSTO 2013"
}

Knowledge of harmful use headphones for music and practices of health hazard en students of the Faculty of Nursing - One, September 2012 - August 2013

Flora Martha Huisacayna Díaz, ${ }^{1, a, c}$, Yolanda Aguilar Semino de Flores ${ }^{2, a}$, Ana Cecilia Flores Garcia $^{3, a, b}$, Redu Yupanqui Cayampi ${ }^{4}$

${ }^{1}$ Facultad de enfermería Universidad Nacional "San Luis Gonzaga" de Ica. Ica, Perú. ${ }^{2}$ Hospital Santa María del Socorro. Ica, Perú.

${ }^{3}$ Hospital Augusto Hernández Mendoza. Ica, Perú. ${ }^{4}$ Estudiante de la Facultad de Enfermería Universidad Nacional San Luis Gonzaga de Ica- Perú.

${ }^{\mathrm{a}}$ Licenciada en Enfermería, ${ }^{b}$ Enfermera Especialista en Pediatría, ${ }^{\circ}$ Magíster en Administración y Planificación de Educación Superior.

\section{RESUMEN}

Objetivo: Determinar el nivel de conocimiento sobre los efectos nocivos del uso de auriculares para escuchar música y el nivel de prácticas de riesgo para la salud, en estudiantes de la Facultad de Enfermería de la Universidad Nacional "San Luis Gonzaga" de Ica. Material y Método: Estudio descriptivo transversal constituido por 220 estudiantes del I al X Ciclo, se utilizó dos cuestionarios validados con 10 reactivos cada uno de opción dicotómicas y múltiples. Resultados: Conocimiento sobre: El límite de tolerancia acústica por la O.M.S., 45,45\% indican a 90 decibeles. Si daña la salud por el uso excesivo de auriculares para música, el 99,09\% refieren no saber. Tipos de auriculares para escuchar música; que causan mayor daño, 54,54\% desconocen, 13,64\% consideran a los auriculares supra auriculares que causan daño para la salud. El $80,45 \%$ refieren que los auriculares a volumen alto no producen sordera prematura. Prácticas de Riesgo: $75 \%$ de los estudiantes refieren escuchar música con volumen alto; $54,54 \%$ escuchan de 5 a más horas por día; $63,64 \%$ acude a sitios de esparcimiento con volumen alto los fines de semana; $95 \%$ refieren usar los auriculares tipo botón para escuchar su música. Conclusiones: El $59,01 \%$ de los estudiantes de la Facultad de Enfermería de la Universidad Nacional "San Luis Gonzaga" de Ica tienen deficiente nivel de conocimiento sobre los efectos nocivos del uso de auriculares para escuchar música y el $62,73 \%$ desarrollan prácticas de riesgo alto para su salud.

Palabras Claves: Conocimiento, efectos nocivos, uso de auriculares.

\section{SUMMARY}

Objective: Determine the level of knowledge about the harmful effects of using headphones to listen to music and the level of risk practices for health, students in the School of Nursing National "San Luis Gonzaga" of Ica University. Material and Methods: A cross consisting of 220 students from first to fifth cycle two validated questionnaires with 10 reagents each dichotomous and multiple choice was used. Results: Knowledge: The stochastic limit of tolerance with acoustics, 45,45\% indicated 90 decibels. If health damage excessive use of headphones for music, $99,09 \%$ reported not knowing Types of headphones to listen to music that cause the most damage, unknown $54,54 \%, 13,64 \%$ above consider headphones headphones that cause damage to health. The $80,45 \%$ reported that the headphones do not produce loud premature deafness. Risk Practices: $75 \%$ of students report hearing loud music; $54,54 \% 5$ to hear more hours per day; $63,64 \%$ goes to recreation sites with high volume weekends; 95\% reported using the button type headphones and hear music. Conclusions: $59,01 \%$ of students in the Faculty of Nursing at the "San Luis Gonzaga" of Ica National University have poor level of knowledge about the harmful effects of using headphones to listen to music and $62,73 \%$ develop high-risk practices for health.

Keywords: Knowledge, adverse effects, wearing headphones. 


\section{INTRODUCCION}

La Organización Mundial de la Salud (OMS) informa que 360 millones de personas en el mundo (328 millones de adultos y 32 millones de menores) presentan pérdida de audición discapacitante, lo que corresponde al $5 \%$ de la población mundial (1). Siendo la lesión sensorial más prevalente a nivel global. Los niveles de ruido se miden en decibeles $(\mathrm{dB})$, continúa refiriendo la OMS que el oído se daña cuando se supera a más de 8 horas de escuchar música con audífonos a un volumen elevado y más de dos horas diarias. La exposición a ruidos muy elevados puede causar perdida de la audición progresiva e irreversible por el deterioro en el nervio auditivo. Cada vez es más común ver a cientos de adolescentes escuchando música a través de los auriculares con su equipo portátil (celular), esta práctica que parece inofensiva puede ser peligrosa en la medida que continúen escuchando música a un volumen elevado, sufrirán una disminución de la capacidad auditiva que se hará más evidente en la edad adulta que les tocará vivir. La potencia de los audífonos del reproductor MP3 puede alcanzar hasta los 100 decibeles de potencia, siendo muy excesivo para el oído humano (2).

Según la OMS la exposición diaria al ruido con auriculares para escuchar música no debe superar más de los 60 decibeles ya que el sonido con los auriculares es proyectado directamente al oído por lo que recomiendan escuchar a un volumen bajo y un máximo de 30 minutos por día, así mismo señala que el ruido causa miles de muertes por año y que la exposición a largo plazo estaría causando los accidentes de tránsito en un $8 \%$ y los ataques cardiacos en un 3.5\% (3). Se estima que 1 de cada 5 personas que escucha música por más de 3 horas con los auriculares y a más de 90 decibeles sufrirá hipoacusia prematura (4). En estudios internacionales de los adolescentes sobre la pérdida auditiva inducida por el ruido en adolescentes (5) concluye: que el $63 \%$ de los estudiantes utilizan los auriculares a un volumen de 80 a 90 decibeles y el tiempo de uso de 6 a más horas con su MP3; el 25\% de los estudiantes tenían cefalea, el $40 \%$ zumbido de oídos, el $35 \%$ son muy distraídos en sus estudios, el $39.5 \%$ tenían ansiedad y sus conocimientos eran bajos en relación a años anteriores por el uso de los auriculares tipo botón (5). Otro estudio titulado "Alerta sobre el daño en los oídos" con una población de 50 estudiantes de una escuela secundaria concluye: el $56 \%$ de estudiantes utilizan auriculares de tipo definido como el más peligroso, los intra auriculares tipo botón, seguido por los supra auriculares en un $30 \%$ los circumaurales y los in-ear con un $16 \%$ que son los menos utilizados, el promedio de horas los intraaurales se escuchan casi 4 horas con un volumen máximo y solo el $36 \%$ a nivel de su potencia. Otro estudio internacional por Sismanis Mariela Felix concluye que el 50\% de estudiantes tienen lesiones auditivas: $33 \%$ leve y un $17 \%$ grave atribuyen las pérdidas de audición a las costumbres de los jóvenes de escuchar música a través de reproductores MP3 con auriculares de botón; el 10\% de pérdida de la capacidad auditiva, afectaron en especial a la captación de sonidos agudos como ocurre a los ancianos que empiezan a sufrir sordera (6). En este contexto, por todos estos considerandos, el objetivo fue determinar el nivel de conocimiento sobre los efectos nocivos del uso de auriculares para escuchar música y el nivel de prácticas de riesgo para la salud, en estudiantes de la Facultad de Enfermería de la Universidad Nacional "San Luis Gonzaga" de Ica.

\section{MATERIAL Y METODO}

El estudio utilizo fue el método descriptivo de enfoque cualitativo y de diseño no experimental de corte transversal. La población de estudiantes de la Facultad de Enfermería estuvo conformada por 535, obteniéndose una muestra de 220 estudiantes con aplicación de la fórmula de población finita, la muestra se distribuyó de 
acuerdo al muestreo aleatorio estratificado mediante la modalidad de afiliación proporcional, ejecutándose en la Ciudad Universitaria en la Facultad de Enfermería a estudiantes del I al X Ciclo. La técnica de recolección de datos fue la encuesta, como instrumento se utilizó dos cuestionarios de opción múltiple y dicotómica, validado por juicio de expertos y coeficiencia $R$ de Pearson según coeficiencia Alfa de Cronbach de $(0,82)$ de confiabilidad. El primer cuestionario incluyo datos demográficos y el nivel de conocimiento sobre los efectos nocivos del uso de auriculares para escuchar música y el segundo sobre las prácticas de riesgo para la salud realizado por los estudiantes. Para el análisis estadístico se utilizó el procesador estadístico SPS versión 18.

\section{RESULTADOS}

Referente al Nivel de Conocimiento: En relación al límite de tolerancia acústica por la OMS, $9,1 \%$ refiere a 60 decibeles, el $10 \%$ indica a 70 decibeles, $18,18 \%$ a 80 decibeles, $45,5 \%$ a 90 decibeles, $9,09 \%$ a 100 decibeles, $8,18 \%$ a más de 100 decibeles. Sobre el uso de auriculares para música que daña la salud, el $99,09 \%$ refieren que no dañan, $30,91 \%$ indican que si dañan. Sobre el tipo de auriculares para escuchar música que producen mayor daño para la salud, el $10.90 \%$ refieren que causan daño los de tipo botón, 9,1\% consideran a los In - ear, 13,64\% a los supra auriculares, $11,82 \%$ a los circumaurales, $54,54 \%$ no saben. Sobre si la música elevada con auriculares y con un tiempo de uso prolongado produce sordera prematura, el $80,45 \%$ responden que no producen, $19,55 \%$ refieren que si producen sordera. Prácticas de Riesgo: Sobre el nivel del volumen que les gusta escuchar música con auriculares, el $75 \%$ escucha a volumen alto, el $15,9 \%$ escuchan a volumen medio, y el $9,1 \%$ escuchan a volumen bajo, sobre el tiempo que escucha música con auriculares diariamente, el $54,54 \%$ escucha de 5 a más horas diarias, $20,45 \%$ escucha 4 horas, $11,37 \%$ tres horas, $9,09 \% 2$ horas y, $4,55 \%$ escucha 1 hora diaria. Si acude a sitios de esparcimiento de volumen alto el fin de semana, $63,64 \%$ refieren ir a veces, el $19 \%$ si escuchan, $17,27 \%$ no acuden. Si gozar de su música preferencial aumenta el volumen, el $50 \%$ refieren que si, $27,28 \%$ refieren que no, $22,72 \%$ refieren que a veces aumentan el volumen.

Tabla 1. Conocimiento sobre los efectos nocivos del uso de auriculares para escuchar música

\begin{tabular}{|c|c|c|}
\hline $\begin{array}{l}\text { El límite de tolerancia acústica } \\
\text { recomendada por la OMS }\end{array}$ & $\mathbf{N}^{\circ}$ & $\%$ \\
\hline 60 Decibeles & 20 & 9,1 \\
\hline $70 \mathrm{~dB}$ & 22 & 10 \\
\hline $80 \mathrm{~dB}$ & 40 & 18,18 \\
\hline $90 \mathrm{~dB}$ & 100 & 45,45 \\
\hline $100 \mathrm{~dB}$ & 20 & 9,09 \\
\hline 100 a más dB & 18 & 8,18 \\
\hline $\begin{array}{l}\text { Sobre si causa daño el uso de auriculares } \\
\text { para escuchar música }\end{array}$ & $\mathrm{N}^{\circ}$ & $\%$ \\
\hline $\begin{array}{l}\text { Si } \\
\text { No }\end{array}$ & $\begin{array}{r}68 \\
152\end{array}$ & $\begin{array}{l}30,91 \\
69,09\end{array}$ \\
\hline $\begin{array}{l}\text { Tipo de auriculares para escuchar música } \\
\text { que causan mayor daño }\end{array}$ & $\mathrm{N}^{\circ}$ & $\%$ \\
\hline Tipo botón & 29 & 10,90 \\
\hline Tipo In - ear & 20 & 9,1 \\
\hline Tipo supra auriculares & 30 & 13,64 \\
\hline Tipo circumaurales & 26 & 11,82 \\
\hline No sabe & 120 & 54,54 \\
\hline
\end{tabular}




\begin{tabular}{lcc}
\hline $\begin{array}{l}\text { Escuchar música elevada con auriculares } \\
\text { y de uso prolongado produce sordera } \\
\text { prematura }\end{array}$ & No & $\%$ \\
Si & 43 & 19,55 \\
No & 177 & 80,45 \\
Escuchar música por tiempo prolongado a & & \\
volumen alto puede producir problemas & & \\
psicológicos & $N^{\circ}$ & $\%$ \\
Dificultad para el sueño & 40 & 18,18 \\
Cefalea & 35 & 15,91 \\
estrés & 30 & 13,63 \\
Disminución de la capacidad auditiva & 25 & 11,36 \\
Dificultad para concentrase & 15 & 6,82 \\
Problemas de memoria & 5 & 2,28 \\
Ninguno & 70 & 31,82 \\
\hline
\end{tabular}

Tabla 2. Prácticas de Riesgo para la salud

\begin{tabular}{|c|c|c|}
\hline $\begin{array}{c}\text { Tipos de auriculares que utiliza para escuchar } \\
\text { música }\end{array}$ & $\mathbf{N}^{\circ}$ & $\%$ \\
\hline Tipo botón & 209 & 95 \\
\hline Tipo supra auriculares & 4,4 & 2 \\
\hline Tipo circumaurales & 4,2 & 2 \\
\hline Tipo In - ear & 2,2 & 1 \\
\hline $\begin{array}{l}\text { Volumen con que escucha música con } \\
\text { auriculares }\end{array}$ & $\mathrm{N}^{\circ}$ & $\%$ \\
\hline Volumen alto & 165 & 75 \\
\hline Volumen medio & 35 & 15,9 \\
\hline Volumen bajo & 20 & 9,1 \\
\hline $\begin{array}{l}\text { Tipo que escucha música con auriculares } \\
\text { diariamente }\end{array}$ & $\mathrm{N}^{0}$ & $\%$ \\
\hline De 5 a más horas & 120 & 54,54 \\
\hline 4 horas & 45 & 20,45 \\
\hline 3 horas & 25 & 11,37 \\
\hline 2 horas & 20 & 9,09 \\
\hline 1 hora & 10 & 4,55 \\
\hline $\begin{array}{l}\text { Acude a sitios de esparcimiento de volumen alto } \\
\text { los fines de semana }\end{array}$ & $\mathrm{N}^{\circ}$ & $\%$ \\
\hline Si & 42 & 19,09 \\
\hline No & 38 & 17,27 \\
\hline A veces & 140 & 63,64 \\
\hline \multicolumn{3}{|l|}{ Para gozar de su música aumenta el volumen } \\
\hline & $\mathrm{N}^{\circ}$ & $\%$ \\
\hline Si & 110 & 50,00 \\
\hline No & 60 & 27,28 \\
\hline A veces & 50 & 22,72 \\
\hline
\end{tabular}

Tabla 3. Datos demográficos

\begin{tabular}{lcc}
\hline Edad & $\mathbf{N}^{\circ}$ & $\%$ \\
\hline $\mathbf{1 6}$ a $\mathbf{2 0}$ años & 140 & 63,6 \\
$\mathbf{2 1}-\mathbf{2 5}$ años & 65 & 29,5 \\
$\mathbf{2 6}-\mathbf{3 0}$ años & 10 & 4,5 \\
$\mathbf{3 1}$ a más años & 5 & 2,4 \\
Sexo & $\mathrm{N}^{\circ}$ & $\%$ \\
Masculino & 32 & 14,5 \\
Femenino & 188 & 85,5 \\
\hline
\end{tabular}




\section{DISCUSIÓN.}

Con respecto al nivel de conocimiento sobre efectos nocivos del uso de auriculares para música nos indican que el 59,10\% (130) tienen deficiente nivel de conocimiento sobre (límite de tolerancia acústica por la OMS: $45,45 \%$ que indican como límite de tolerancia a 90 decibeles, $18,18 \%$ a 80 decibeles; si causan daño el uso de auriculares para escuchar música, 69,09\% indican que no, el $30,91 \%$ indican que si causan daño; tipos de auriculares que causan mayor daño, 13,64\% refieren a los supra auriculares, 11,82\% circumaurales, $10,90 \%$ al tipo botón; escuchar música elevada con auriculares y por uso prolongado producen sordera prematura, $80,45 \%$ indican que no, $19,55 \%$ refieren que sí) y el 40,90\% (90) tienen eficiente nivel de conocimiento. Estos resultados difieren de BLOJ- Lia (2) que refiere que el $80 \%$ en los jóvenes conocen los efectos nocivos de los ruidos elevados y el $88 \%$ de ellos a la pérdida auditiva. Similares resultados se encontró al estudio realizado en el Instituto Real para Sordos (7), quienes indican que el 59\% no sabían que el daño podía ser permanente e irreversible, el $80 \%$ de los participantes sabían que un volumen alto es perjudicial para la audición, pero solo la mitad de ellos sabían que el tiempo de exposición afecta también a la hora de sufrir un daño, otro estudio por Rodríguez Dolores que refiere que el $45 \%$ de los jóvenes poseen regular nivel de conocimiento sobre los efectos nocivos del ruido, $26 \%$ con suficiente nivel de conocimiento, $28 \%$ no sabe sobre el tema y el $1 \%$ tiene buen nivel de conocimiento (8).

Respecto a las prácticas de riesgo para la salud: El estudio refiere que el $62,74 \%$ (138) estudiantes realizan prácticas de riesgo alto por: (utilizar auriculares tipo botón en un 95\% (209; escuchar música elevada con auriculares a un volumen alto en un $75 \%$; el $54,54 \%$ escucha música con audífonos de 5 a 6 horas diarias, el $63,64 \%$ a veces acude a sitios de esparcimiento con volumen alto, el $19,09 \%$ si acuden a los sitios de esparcimiento; el $50 \%$ aumenta el volumen para gozar de la música); el 29,09\% (64) realiza prácticas de riesgo inadecuadas y el $8,18 \%$ realiza prácticas de riesgo bajo. Igual el resultado se encontraron por Colombo, Marina (9) que refiere el $50 \%$ de los jóvenes encuestados poseen hábitos medianamente perjudicables, el $58 \%$ tienen hábitos de escuchar música a elevada intensidad, el $50 \%$ asiste de 2 a 3 veces por mes a lugares con música de elevada intensidad el $38 \%$ lo hacen una vez al mes y el $60 \%$ escuchan música de 1 a 4 horas diarias.

\section{CONCLUSIONES}

EL 59,01\% de los estudiantes de la Facultad de Enfermería de la Universidad Nacional "San Luis Gonzaga" de Ica tienen deficiente nivel de conocimiento sobre los efectos nocivos del uso de auriculares para escuchar música y el $62,73 \%$ desarrollan prácticas de riesgo alto para la salud.

\section{Correspondencia:}

Mag. Flora Martha Huisacayna Díaz

Correo electrónico: martha.fhd@hotmail.com

\section{REFERENCIAS BIBLIOGRAFICAS}

1. Organización Mundial de la Salud (OMS) 2010 "Decibeles - sordera prematura/ España. (citado 20 de mayo 2013). Disponible en htp//www.who.in/ topies/decibeles

2. Bloj, Lia. Contaminación Sonora entre adolescentes en http://www.diarioC.com. $\mathrm{ar} /$ tecnologa/contaminacion sonora entre adolescentes/100802/17 de agosto del 2012.

3. Organización Mundial de la Salud (OMS): Convenio Marco de la OMS para evitar sordera prematura contaminación acústica 2006- p. 74.

4. Kogan, C; Carlos M. Sosa. Evaluación de los hábitos de uso de los reproductores portátiles de música adolescente. Primera Jornada Regional Acústica ADAD 2009 - Rosario p. 104. 
5. Lía, A. Del Prado España. Alerta sobre daños en los oídos. Doctora en Ciencias de la Información Universidad Computence de Madrid, España. 2010. p. 74

6. Sismanis, M. Uso de potentes reproductores MP3 crean una generación sorda. Doctora en Psicología Universidad Computence de Madrid- España. 2010. p. 14.

7. Instituto Nacional de la Sordera y Otros trastornos de la comunicación (NIDCD). El mundo es ruidoso. Disponible en: http:/www.noisyplanet.nided.nihn.gob/site collection.documents/españolhojainforma tiva.PDF Enero 2009- p. 127.
8. Rodríguez C. el conocimiento de los jóvenes sobre los efectos nocivos del ruido. Tesis para Licenciatura en fonoaudiología. Facultad de Ciencias de la Salud Universidad FASTA- Noviembre 2010 - p. 47.

9. Colombo M. "Resultado de estudios audiometricos y hábitos auditivos en jóvenes universitarios" Rosario Tesis para Licenciatura en fonoaudiología Universidad Nacional del Rosario Argentina 2012 - p. 52.

Recibido: 13/02/14

Aprobado para publicación: 05/06/14 